\title{
Amenorrhea, CTCAE
}

National Cancer Institute

\section{Source}

National Cancer Institute. Amenorrhea, CT CAE. NCI Thesaurus. Code C143240.

A disorder characterized by the abnormal absence of menses for at least three

consecutive menstrual cycles 